\title{
NESTED SYMMETRIC REPRESENTATION OF ELEMENTS OF THE SUZUKI CHAIN GROUPS
}

\author{
MOHAMED SAYED
}

Received 2 December 2002

\begin{abstract}
We demonstrate an economic and concise method for representing the elements of groups involved in the Suzuki chain. For example, we represent each element of Suz : 2 by a permutation on 14 letters from $L_{3}(2): 2$ followed by four words, each of length at most two, in 14, 36, 100, and 416 involutory symmetric generators, respectively. Such expressions will have an obvious advantage over permutations on 1782 provided that it is reasonably simple to multiply and invert them. We refer to this as nested symmetric representation of an element of the group.
\end{abstract}

2000 Mathematics Subject Classification: 20B40, 20D60.

1. Introduction. An element of order three in the class $3 D$ (see [2]) of Conway's group $\mathrm{Co}_{1}$ is centralized by $3 \times A_{9}$ and the chain of subgroups $K_{i}$ (of $\mathrm{Co}_{1}$ ) which are the centralizers of the subgroups $A_{i}$ obtained by fixing all but $i$ points in this $A_{9}$ is called Suzuki chain, see [2, 8, 9]. This chain of subgroups in $\mathrm{Co}_{1}$, discovered by Thompson (unpublished), see [6], has been of interest in finite groups.

In the present work, each of the Suzuki chain groups emerges as a group $G$ generated by a set of $\left|\left(K_{i}: 2\right):\left(K_{i+1}: 2\right)\right|$ involutions whose set normalizer in $G$ is isomorphic to $K_{i}: 2$. Most of these groups are constructed by hand using the double coset enumeration technique shown in [5].

The main purpose of this paper is to introduce the concept of nested symmetric representation of elements of a group. In general, if we wish to multiply and invert elements in a straightforward manner, we must represent them as either permutations or matrices. The two operations are particularly easy to perform on permutations. Moreover, the cycle shape of an element immediately yields its order, and often its conjugacy class. However, for large sporadic groups, the lowest degree of permutation representations are unmanageable. Operations on matrices are much more difficult and basic information about an element is not readily recovered from its matrix representation. The approach illustrated in this paper combines conciseness with acceptable ease of manipulation and makes hand calculations with the elements possible. Inversion and multiplication can be performed manually or mechanically [1] by means of short recursive algorithms. 
2. Involutory symmetric generators of groups. Let $G$ be a group and let $T=\left\{t_{0}, t_{1}, \ldots, t_{n-1}\right\}$ be a set of elements of order $m$ in $G$. Making the definitions $T_{i}=\left\langle t_{i}\right\rangle$ and $\bar{T}=\left\{T_{0}, T_{1}, \ldots, T_{n-1}\right\}$ allows us to define $N=\mathcal{N}_{G}(\bar{T})$, the set normalizer in $G$ of $\bar{T}$. We say that $T$ is a symmetric generating set for $G$ if the following two conditions hold:

(i) $G=\langle T\rangle$,

(ii) $N$ permutes $\bar{T}$ transitively.

We call $N$ the control subgroup. Conditions (i) and (ii) imply that $G$ is a homomorphic image of the progenitor

$$
m^{* n}: N
$$

where $m^{* n}$ represents a free product of $n$ copies of the cyclic group $C_{m}$ and $N$ is a group of automorphisms of $m^{* n}$ which permutes the $n$ cyclic subgroups by conjugation, see $[3,4,5]$.

Since in this paper we are only concerned with involutory symmetric generators, we restrict our attention to the case $m=2$ (while $N$ will simply act by conjugation as permutations of the $n$ involutory symmetric generators).

THEOREM 2.1. All non-abelian finite simple groups can arise as finite homomorphic images of progenitors of the form $2^{* n}: N$.

Proof. Let $H$ be a maximal subgroup of a finite simple group $G$. Suppose that $1 \neq \mathbf{t} \in G, \mathbf{t}^{2}=1$. Under the subgroup $H, \mathbf{t}^{G}$, the conjugacy class of $\mathbf{t}$ in $G$, splits into orbits as

$$
\mathbf{t}^{G}=\mathscr{T}_{1} \dot{\cup} \mathscr{T}_{2} \dot{\cup} \cdots \dot{\cup} \mathscr{T}_{r}
$$

Without loss of generality, we may assume that $\mathscr{T}_{1}=\left\{\mathbf{t}_{0}, \mathbf{t}_{1}, \ldots, \mathbf{t}_{n-1}\right\}$ is not a subset of $H$. It is clear that

$$
\mathcal{N}_{G}\left(\left\langle\mathscr{T}_{1}\right\rangle\right) \geq\left\langle H, \mathscr{T}_{1}\right\rangle=G
$$

since $H$ is maximal in $G$ and $\mathscr{T}_{1}$ is not a subset of $H$. Therefore,

$$
1 \neq\left\langle\mathscr{T}_{1}\right\rangle \triangleleft G,
$$

and, since $G$ is simple, we have

$$
\left\langle\mathscr{T}_{1}\right\rangle=G
$$

Moreover, if $\pi \in H$ and $\mathbf{t}_{i}^{\pi}=\mathbf{t}_{i}(i=0,1, \ldots, n-1)$, then $\pi \in \mathscr{L}(G)$ and so $\pi=1$, that is, $H$ permutes the elements of $\mathscr{T}_{1}$ faithfully (and transitively). Now, let $2^{* n}$ denote a free product of $n$ copies of the cyclic group $C_{2}$ with involutory generators $t_{0}, t_{1}, \ldots, t_{n-1}$ and let $N \cong H$ consist of all automorphisms of $2 * n$ which permute the $t_{i}$ as $H$ permutes the $\mathbf{t}_{i}$ :

$$
\pi^{-1} t_{i} \pi=t_{i}^{\pi}=t_{\pi(i)} \quad \text { for } \pi \in N .
$$


Then, clearly $G$ is a homomorphic image of $2^{* n}: N$, a split extension of $2^{* n}$ by the permutation automorphisms $N$.

Since the progenitor is a semidirect product (of $\langle T\rangle$ with $N$ ), it follows that, in any homomorphic image $G$, we may use the equation

$$
t_{i} \pi=\pi t_{i}^{\pi}=\pi t_{\pi(i)}
$$

or $i \pi=\pi i^{\pi}$ as we will more commonly write (see below) to gather the elements of $N$ over to the left. Another consequence of this is that a relation of the form $\left(\pi t_{i}\right)^{n}=1$ for some $\pi \in N$ in a permutation progenitor becomes

$$
\pi^{n}=t_{i} t_{\pi(i)} \cdots t_{\pi^{n-1}(i)}
$$

Each element of the progenitor can be represented as $\pi w$, where $\pi \in N$ and $w$ is a word in the symmetric generators. Indeed, this representation is unique provided that $w$ is simplified so that those adjacent symmetric generators are distinct. Thus any additional relator by which we must factor the progenitor to obtain $G$ must have the form $\pi w\left(t_{0}, t_{1}, \ldots, t_{n-1}\right)$, where $\pi \in N$ and $w$ is a word in $T$.

Now, if $N x N$ is a double coset of $N$ in $G$, we have

$$
N x N=N \pi w N=N w N,
$$

where $x=\pi w \in G$, with $\pi \in N$, and $w$ a word in the symmetric generators. We denote this double coset by $[w]$; for example, [01] denotes the double coset $N t_{0} t_{1} N$. The double coset $N e N=N$, where $e$ is the identity element, is denoted by $[*]$.

NotAtion 2.2. We will allow $i$ to stand for the coset $N t_{i}$, $i j$ for the coset $N t_{i} t_{j}$, and so on. We will also let $i$ stand for the symmetric generator $t_{i}$ when there is no danger of confusion. Thus we write, for instance, $i j \sim k$ to mean $N t_{i} t_{j}=N t_{k}$ and $i j=k$ to mean $t_{i} t_{j}=t_{k}$.

We define the subgroups $N^{i}, N^{i j}, N^{i j k}, \ldots$ (for $i, j$, and $k$ distinct) as follows:

$$
N^{i}=\mathscr{C}_{N}\left(\left\langle t_{i}\right\rangle\right), \quad N^{i j}=\mathscr{C}_{N}\left(\left\langle t_{i}, t_{j}\right\rangle\right), \quad N^{i j k}=\mathscr{C}_{N}\left(\left\langle t_{i}, t_{j}, t_{k}\right\rangle\right)
$$

or, more generally,

$$
N^{i_{1} i_{2} \cdots i_{m}}=\mathscr{C}_{N}\left(\left\langle t_{i_{1}}, t_{i_{2}}, \ldots, t_{i_{m}}\right\rangle\right)
$$

for $i_{1}, i_{2}, \ldots, i_{m}$ distinct.

Let $g$ be an element of $G$. Then we define

$$
N^{(g)}=\{\pi \in N \mid N g \pi=N g\},
$$


where $N^{(g)}$ is called the coset stabilizing subgroup (of $N g$ in $N$ ). Clearly, $N^{w} \leq$ $N^{(w)}$ for $w$ being a word in the symmetric generators and the number of cosets in the double coset $[w]=N w N$ is given by $|N| /\left|N^{(w)}\right|$, see $[5,7]$.

LEMMA 2.3. In $2^{*} n: N,\left\langle t_{i}, t_{j}\right\rangle \cap N \leq \mathscr{C}_{N}\left(N^{i j}\right)$.

Proof. Suppose that some element of $G$ is in $\left\langle t_{i}, t_{j}\right\rangle$ and $N$; then it must centralize everything in $N^{i j}$-since, by definition, everything in $N^{i j}$ commutes with everything in $\left\langle t_{i}, t_{j}\right\rangle$-and also in $N$, see [4]. Of course, this result can be readily generalized to more than two symmetric generators, and the general result is

$$
\left\langle t_{i_{1}}, t_{i_{2}}, \ldots, t_{i_{m}}\right\rangle \cap N \leq \mathscr{C}_{N}\left(N^{i_{1} i_{2} \cdots i_{m}}\right) .
$$

LEMMA 2.4. In $\left(2^{n}: N\right) /\left(\pi_{a b}=a b a\right)$ ( $a b$ is called a special pair),

$$
N^{(i j)} \geq\left\langle N^{i j}, \pi_{k j} \text { with } i k \text { and } k j \text { special pairs }\right\rangle .
$$

Proof. Since $i j \sim i k \cdot k j \sim i \cdot \pi_{k j} \cdot k \sim i^{\pi_{k j}} \cdot k=(i j)^{\pi_{k j}}$, then $\pi_{k j}$ fixes the coset $i j$. Then $\pi_{k j} \in N^{(i j)}$.

3. Manual double coset enumeration. It is now clear that we intend to take our progenitor of shape $2^{* n}: N$, where $N$ is a transitive permutation group on $n$ letters. A canonical presentation for this progenitor is

$$
\left\langle x, y, t \mid\langle x, y\rangle \cong N, t^{2}=1=\left[N^{0}, t\right]\right\rangle,
$$

where $x$ and $y$ generate $N$, and $t$ corresponds to $t_{0}$. The meaning of $1=\left[N^{0}, t\right]$ is that we adjoin the relations $1=\left[x_{1}, t\right]=\left[x_{2}, t\right]=\cdots=\left[x_{p}, t\right]$, where $x_{1}$, $x_{2}, \ldots, x_{p}$ generate $N^{0}$, and $\langle x, y\rangle \cong N$ means that we adjoin sufficient relations between $x$ and $y$ to define $N$.

3.1. The progenitor $2^{*(7+7)}:\left(L_{3}(2): 2\right)$. A presentation for the progenitor is

$$
\left\langle x, y, s \mid x^{2}=y^{3}=(x y)^{8}=[x, y]^{4}=s^{2}=[s, y]=\left[s,[x, y]^{2}\right]=1\right\rangle,
$$

where the action of the elements of the control subgroup $N \cong L_{3}(2): 2$ on the 14 symmetric generators may be given by $x=(0,0)(1,1)(2,2)(3,3)(4,4)(5,5)(6,6)$ and $y=(0,5,6)(1,2,4)(1,6,5)(2,4,3)$. Here the symmetric generators are denoted by seven points $0,1,2,3,4,5$, and 6 , and seven lines $0,1,2,3,4,5$, and 6 in the projective plane shown in Figure 3.1. In order to obtain a finite homomorphic image of such a progenitor, we must factor by some additional relations. There are three two-point stabilizers $N^{i j}$, depending on whether $i$ is a point and $j$ is a line not through it, $i$ is a point and $j$ is a line through it, or $i$ and $j$ are two different points or two different lines. Now consider the first case

$$
N^{00}=\langle(2,4)(5,6)(2,4)(5,6),(1,2)(3,6)(1,2)(3,6)\rangle \cong S_{3},
$$




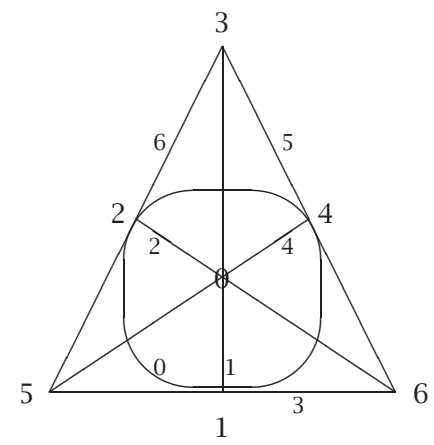

FIGURE 3.1. Seven-point projective plane.

which is centralized by the involution $\pi_{00}=(0,0)(1,1)(2,2)(3,3)(4,4)(5,5)(6,6)$. Lemma 2.3 stated that $\pi_{00}$ is the only permutation of $N$ which can be written in terms of $s_{0}$ and $s_{0}$. We make the assumption that $\pi_{00}=s_{0} s_{0} s_{0}$, a word in the symmetric generators $s_{0}$ and $s_{0}$ of the shortest length that does not lead to collapse. Also

$$
\begin{aligned}
N^{65}= & \langle(0,1)(2,5)(0,4)(2,3),(0,2)(1,5)(0,4)(1,6), \\
& (0,1,2,5)(3,4)(0,6,4,1)(2,3)\rangle \\
\cong & D_{8},
\end{aligned}
$$

which is centralized by the involution $\pi_{65}=(0,2)(1,5)(0,4)(1,6)$. If we can write the involution $\pi_{65}$ as a word in the symmetric generators $s_{6}$ and $s_{5}$, the shortest possibility is $\pi_{65}=\left(s_{6} s_{5}\right)^{2}$. The third relation is $\pi_{01}=\left(s_{0} s_{1}\right)^{2}$, where $\pi_{01}=(2,5)(4,6)(0,3)(2,4) \in \mathscr{C}_{N}\left(N^{01}\right)$.

Consider the group

$$
G \cong \frac{2^{*(7+7)}:\left(L_{3}(2): 2\right)}{\pi_{00}=s_{0} s_{0} s_{0}, \pi_{65}=\left(s_{6} s_{5}\right)^{2}},
$$

from which a simple presentation follows:

$$
\begin{aligned}
\langle x, y, s| x^{2} & =y^{3}=(x y)^{8}=[x, y]^{4}=s^{2}=[s, y] \\
& \left.=\left[s,[x, y]^{2}\right]=(s x)^{3}=\left(x y s^{x y x}\right)^{4}=1\right\rangle .
\end{aligned}
$$

We are now in a position to carry out the double coset enumeration of $G$ over $N$. The set of all double cosets $[w]=N w N$, the coset stabilizing subgroups $N^{(w)}$, and the number of single cosets each contains are shown in Table 3.1. The double coset enumeration shows that the group defined by the symmetric presentation contains a homomorphic image of $L_{3}(2): 2$ to index at most 36, and gives a convenient name to each of the 36 cosets in terms of 14 symmetric generators. Moreover, the action of the generators on the 36 cosets, by right 
TABLE 3.1. Double coset enumeration for $U_{3}(3): 2$.

\begin{tabular}{l|l|c}
\hline Label $[w]$ & Coset stabilizing subgroup $N^{(w)}$ & No. of cosets \\
\hline$[*]$ & $N$ & 1 \\
\hline$[0]$ & $N^{0}=N^{(0)} \cong S_{4}$, & 14 \\
& with orbits $1+6+3+4$ on the 14 points & \\
\hline$[00]=[0]$ & Since $000 \sim * \Rightarrow 00 \sim 0$ & \\
\hline & $N^{01} \cong V_{4} \cdot$ & \\
& Since $01 \sim 05 \cdot 51 \sim 05 \cdot \pi_{15} \cdot 5 \sim 61 \cdot 5 \sim 65$, & \\
& $\pi_{15}=(0,6)(1,5)(2,4)(3,1)(4,0)(5,2)(6,3)$ & \\
& $\Rightarrow N^{(01)} \geq\langle(2,4)(5,6)(2,4)(5,6), \rho\rangle \cong D_{12}$, & \\
& $\rho=(0,6,1,5)(3,1)(2,0,4,4,5,3,6,2)$, & \\
& with orbits $2+4+8$ on the 14 points & \\
\hline 01$]$ & $01 \sim 06 \cdot 61 \sim 06 \cdot 616 \cdot 6 \sim 06 \cdot \pi_{61} \cdot 6$ & \\
& $\sim 216 \sim 1 \cdot 26 \sim 46 \cdot 2 \sim 42 \sim 13 \sim 31$, & \\
& $\pi_{61}=(0,2)(1,3)(2,4)(3,5)(4,6)(5,0)(6,1)$ & \\
\hline 01$]=[01]$ & $012 \sim 312 \sim 3 \cdot 121 \cdot 1 \sim 3 \cdot \pi_{21} \cdot 1 \sim 61 \sim 46$, & \\
& $\pi_{21}=(0,2)(1,0)(2,1)(3,6)(4,3)(5,5)(6,4)$. & \\
& Also $31 \sim 36 \cdot 61 \sim 36 \cdot 616 \cdot 6 \sim 36 \cdot \pi_{61} \cdot 6$ & \\
& $\sim 516 \sim 1 \cdot 56 \sim 66 \cdot 5 \sim 65 \sim 56 \sim 01 \sim 10$ & \\
& &
\end{tabular}

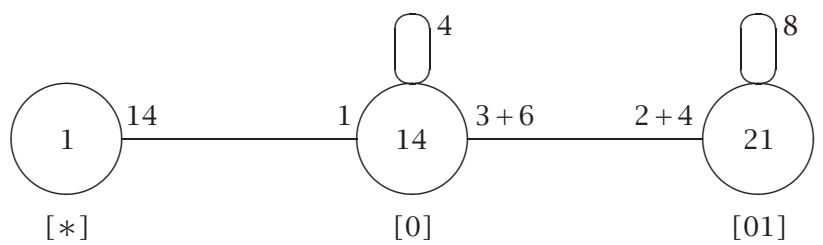

FIGURE 3.2

multiplication, is implicit in the enumeration and so it is readily checked that these permutations satisfy the given relations. Thus, $|G: N| \leq 36$, so $|G| \leq$ $12096=\left|U_{3}(3): 2\right|$, and the (relatively) easy task of finding generators for $U_{3}(3)$ : 2 satisfying the required relations completes the identification of $G$ with $U_{3}(3): 2$. Table 3.1 shows that the Cayley graph of $G$ over $N$ has the form shown in Figure 3.2.

We conclude our work on $G$ by giving symmetrically represented generators for each of the maximal subgroups of: $G \cong\langle(0,0)(1,1)(2,2)(3,3)(4,4)(5,5)(6,6)$, $\left.(0,5,6)(1,2,4)(1,6,5)(2,4,3), s_{0}\right\rangle: U_{3}(3) \cong\left\langle(0,0)(1,1)(2,2)(3,3)(4,4)(5,5)(6,6) s_{0}\right.$, $(0,5,6)(1,2,4)(1,6,5)(2,4,3)\rangle$ is a simple subgroup of index 2 in $G$;

$$
\begin{array}{r}
3_{+}^{1+2}: 8: 2 \cong\langle(0,0)(1,1)(2,2)(3,3)(4,4)(5,5)(6,6), \\
\left.(0,2,2,0,4,5,3,1)(1,4,6,6)(5,3) s_{6} s_{3}\right\rangle
\end{array}
$$


is a subgroup of order 432 with index 28 in $G$ and is the normalizer of

$$
(0,0)(1,1)(2,2)(3,3)(4,4)(5,5)(6,6) s_{6},
$$

an element of order 3 in class $3 A$;

$$
\begin{gathered}
L_{3}(2): 2 \cong\langle(0,0)(1,1)(2,2)(3,3)(4,4)(5,5)(6,6), \\
(0,5,6)(1,2,4)(1,6,5)(2,4,3)\rangle
\end{gathered}
$$

is a subgroup of order 336 with index 36 in $G$ and is the stabilizer of a point in the 36-point graph; and

$$
4 \cdot S_{4}: 2 \cong\left\langle s_{0},(2,6)(4,5)(0,3)(5,6) s_{1} s_{0}\right\rangle
$$

is a subgroup of order 192 with index 63 in $G$ and is the centralizer of

$$
(2,4)(5,6)(2,4)(5,6) \text {, }
$$

an involution in class $2 A$.

3.2. The progenitor $2 * 36:\left(U_{3}(3): 2\right)$. A presentation for the progenitor is

$$
\begin{aligned}
\langle x, y, s, r| x^{2} & =y^{3}=(x y)^{8}=[x, y]^{4}=s^{2}=[s, y]=\left[s,[x, y]^{2}\right] \\
& \left.=(x s)^{3}=\left(x y s^{x y x}\right)^{4}=r^{2}=[r, x]=[r, y]=1\right\rangle .
\end{aligned}
$$

Here the symmetric generators are labeled with the vertices of the 36-point graph as follows. A single vertex is labeled $\infty, 14$ vertices are labeled $0,1,2,3,4$, $5,6,0,1,2,3,4,5$, and 6 , and the 21 vertices are labeled by the 21 flags (a point with a line through it) $01,02,04,10,11,13,20,22,26,31,35,36,40,44,45,53,54,56$, 62,63 , and 65 . We would like to know which elements of the control subgroup $N \cong U_{3}(3): 2$ can be written in terms of two symmetric generators. Lemma 2.3 says that $\mathscr{C}_{N}\left(N^{\infty 0}\right)=\langle s\rangle$. We make the assumption that $\pi_{\infty 0}(=s)=r_{\infty} r_{0} r_{\infty}$, a word in the symmetric generators $r_{\infty}$ and $r_{0}$ of the shortest length that does not lead to collapse.

Consider the group

$$
G \cong \frac{2^{* 36}:\left(U_{3}(3): 2\right)}{\pi_{\infty 0}=r_{\infty} r_{0} r_{\infty}}
$$

from which a simple presentation follows:

$$
\begin{aligned}
\langle x, y, s, r| x^{2} & =y^{3}=(x y)^{8}=[x, y]^{4}=s^{2}=[s, y]=\left[s,[x, y]^{2}\right] \\
& \left.=(x s)^{3}=\left(x y s^{x y x}\right)^{4}=r^{2}=[r, x]=[r, y]=(s r)^{3}=1\right\rangle .
\end{aligned}
$$

The double cosets and coset stabilizing subgroups are shown in Table 3.2. The double coset enumeration yields a Cayley diagram of $G$ over $N$ (see Figure 3.3). 
TABLE 3.2. Double coset enumeration for $J_{2}: 2$.

\begin{tabular}{|c|c|c|}
\hline Label $[w]$ & Coset stabilizing subgroup $N^{(w)}$ & No. of cosets \\
\hline$[*]$ & $N$ & 1 \\
\hline$[\infty]$ & $\begin{array}{l}N^{\infty}=N^{(\infty)} \cong L_{3}(2): 2, \\
\text { with orbits } 1+14+21 \text { on the } 36 \text { points }\end{array}$ & 36 \\
\hline$[\infty 0]=[\infty]$ & Since $\infty 0 \infty \sim * \Rightarrow \infty 0 \sim \infty$ & \\
\hline$[\infty 11]$ & $\begin{array}{l}N^{\infty 11}=\langle(0,0)(1,1)(2,2)(3,3)(4,4)(5,5)(6,6),(0,3) \\
(2,5,4,6)(0,3)(2,6,4,5),(2,4)(5,6)(0,3)(2,4)\rangle \cong D_{16} . \\
\text { Each element } i \text { of the }(2+4) \text {-orbits of } D_{16} \text { is joined } \\
\text { to both } \infty \text { and } 11, \text { that is, } \infty i \text { and } i 11 \text { are special } \\
\text { pairs } \Rightarrow N^{(\infty 11)} \geq\left\langle\pi_{011}, \pi_{111}, \pi_{311}, \pi_{011}, \pi_{111}, \pi_{311},\right. \\
(0,0)(1,1)(2,2)(3,3)(4,4)(5,5)(6,6),(2,4)(5,6)(0,3) \\
(2,4),(0,3)(2,5,4,6)(0,3)(2,6,4,5)\rangle \cong 4 \cdot S_{4}: 2, \\
\pi_{011}=s_{0} s_{3} s_{0}=(2,4)(5,6)(2,4)(5,6) s_{3}, \\
\pi_{111}=s_{1} s_{1} s_{1}=(2,4)(5,6)(2,4)(5,6) s_{1}, \\
\pi_{311}=s_{3} s_{0} s_{3}=(2,4)(5,6)(2,4)(5,6) s_{0}, \\
\pi_{011}=s_{0} s_{3} s_{0}=(2,4)(5,6)(2,4)(5,6) s_{3}, \\
\pi_{111}=s_{1} s_{1} s_{1}=(2,4)(5,6)(2,4)(5,6) s_{1}, \\
\pi_{311}=s_{3} s_{0} s_{3}=(2,4)(5,6)(2,4)(5,6) s_{0}, \\
\text { with orbits } 12+24 \text { on the } 36 \text { points }\end{array}$ & 63 \\
\hline$[\infty 112]=[\infty 11]$ & $\begin{array}{l}r_{\infty} r_{11} r_{2}=r_{11} r_{\infty} r_{2}=r_{11} \cdot \pi_{\infty 2} \cdot r_{\infty}=r_{11} s_{2} r_{\infty} \\
\sim r_{13} r_{\infty} \text { since } 11 \cdot 2 \sim 112 \sim 1 \cdot 1212 \cdot 21 \\
\sim 1 \cdot(0,5)(3,6)(1,3)(2,6) \cdot 21 \sim 321 \sim 31\end{array}$ & \\
\hline
\end{tabular}

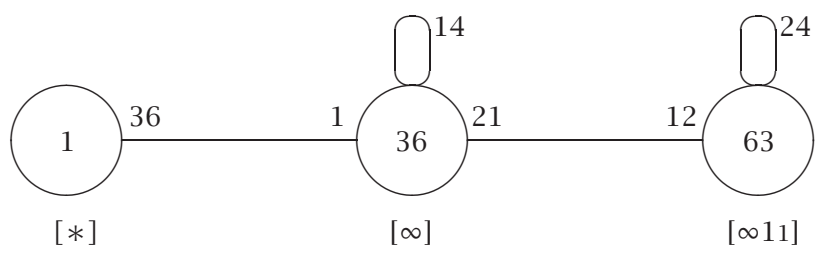

FIGURE 3.3

One should note that the graph obtained above is not the regular graph, but the Cayley one. The regular graph whose automorphism group is $G$ is obtained from the above one by joining the coset $w$ to the coset $i w$. We may readily construct our symmetric generators as permutations of $1+36+63=100$ letters and verify that they do indeed satisfy the relations we assumed, thus proving that the group $G$ has order $12096 \times 100=1209600=\left|J_{2}: 2\right|$. Identifying $G$ with $J_{2}: 2$ is straightforward and follows immediately from the construction of $J_{2}: 2$ as the automorphism group of a rank-3 graph on 100 points, with suborbits 1,36 , and 63 , and point stabilizer $U_{3}(3): 2$, see [2]. 
Every element of $G$ can be represented by a permutation on 36 letters followed by a word in the symmetric generators of length at most two. Alternatively and more concisely, we can represent each element of $G$ by an expression of the form $\pi u v$, where $\pi$ is a permutation on 14 letters (element of $L_{3}(2): 2$ ) and $u$ and $v$ are words of length at most two in the symmetric generators $s$ 's and $r$ 's, respectively. The nested symmetrical representation of generators for each of the maximal subgroups of $G \cong\langle(0,0)(1,1)(2,2)(3,3)(4,4)(5,5)(6,6)$, $\left.(0,5,6)(1,2,4)(1,6,5)(2,4,3), s_{0}, r_{\infty}\right\rangle$

$$
\begin{gathered}
J_{2} \cong\left\langle(0,0)(1,1)(2,2)(3,3)(4,4)(5,5)(6,6) s_{0},(0,5,6)(1,2,4)(1,6,5)(2,4,3),\right. \\
\left.(0,0)(1,1)(2,2)(3,3)(4,4)(5,5)(6,6) r_{\infty}\right\rangle
\end{gathered}
$$

is a simple subgroup of index 2 in $G$;

$$
\begin{gathered}
U_{3}(3): 2 \cong\langle(0,0)(1,1)(2,2)(3,3)(4,4)(5,5)(6,6), \\
\left.(0,5,6)(1,2,4)(1,6,5)(2,4,3), s_{0}\right\rangle
\end{gathered}
$$

is a subgroup of order 12096 with index 100 in $G$ and is the stabilizer of a point in the 100-point graph;

$$
3 \cdot A_{6} \cdot 2^{2} \cong\left\langle(0,3)(1,1)(2,5)(3,0)(4,6)(5,4)(6,2) s_{6} s_{2} r_{3} r_{02}, s_{0}\right\rangle
$$

is a subgroup of order 4320 with index 280 in $G$ and is the normalizer of

$$
(0,3)(1,1)(2,6)(3,0)(4,5)(5,2)(6,4) s_{3},
$$

an element of order 3 in class $3 A$;

$$
\begin{gathered}
2_{-}^{1+4}: S_{5} \cong\left\langle(0,0)(1,1)(2,2)(3,3)(4,4)(5,5)(6,6) s_{0},\right. \\
\left.(0,3)(5,6)(2,6)(4,5) s_{3} s_{1} r_{\infty}\right\rangle
\end{gathered}
$$

is a subgroup of order 3840 with index 315 in $G$ and is the normalizer of

$$
(2,4)(5,6)(2,4)(5,6) \text {, }
$$

an involution in class $2 A$;

$$
\begin{aligned}
2^{2+4} & :\left(3 \times S_{3}\right): 2 \\
& \cong\left\langle(0,5,6)(1,2,4)(1,6,5)(2,4,3),(1,2)(3,6)(1,2)(3,6), s_{0}, r_{1}\right\rangle
\end{aligned}
$$

is a subgroup of order 2304 with index 525 in $G$ and is the normalizer of

$$
\langle(0,5)(3,6)(1,3)(2,6),(0,6)(3,5)(1,3)(4,5)\rangle,
$$

a four-group whose involutions are in class $2 A$;

$$
\begin{gathered}
\left(A_{4} \times A_{5}\right): 2 \cong\left\langle(0,0)(1,4)(2,2)(3,5)(4,1)(5,3)(6,6) s_{6} r_{6},\right. \\
\left.(1,2)(3,6)(1,2)(3,6) s_{0} r_{\infty}\right\rangle
\end{gathered}
$$


is a subgroup of order 1440 with index 840 in $G$;

$$
\begin{aligned}
\left(A_{5} \times D_{10}\right) \cdot 2 \cong\left\langle(0,0,3,4,2,1,4,6)(1,5,5,2)(6,3) s_{6} \mathcal{S}_{5},\right. \\
\\
\left.(0,1)(1,2,3,4)(2,5,5,0,6,6,4,3) s_{6} r_{3} r_{53}\right\rangle
\end{aligned}
$$

is a subgroup of order 1200 with index 1008 in $G$ and is the normalizer of

$$
(0,2,6)(1,3,5)(0,5,4)(1,6,3) s_{2} s_{2} r_{4} r_{36},
$$

an element of order 5 in class $5 A$;

$$
\begin{aligned}
L_{3}(2): 2 \times 2 \cong & \langle(0,0)(1,1)(2,2)(3,3)(4,4)(5,5)(6,6), \\
& \left.(0,5,6)(1,2,4)(1,6,5)(2,4,3), r_{\infty}\right\rangle
\end{aligned}
$$

is a subgroup of order 672 with index 1800 in $G$ and is the centralizer of $r_{\infty}$, an involution in class $2 C$;

$$
\begin{aligned}
5^{2}:\left(4 \times S_{3}\right) \cong\langle & (0,6,5,4,3,2,1)(0,1,2,3,4,5,6) s_{4} r_{0}, \\
& (0,3,2)(1,5,6)(0,4,5)(1,6,2) s_{6} s_{3}, \\
& \left.(0,6)(1,3)(2,2)(3,4)(4,5)(5,1)(6,0) s_{6}\right\rangle
\end{aligned}
$$

is a subgroup of order 600 with index 2016 in $G$; and

$$
S_{5} \cong\left\langle(0,2,6)(1,3,5)(0,5,4)(1,6,3) s_{2} s_{2} r_{4} r_{36}, r_{\infty}\right\rangle
$$

is a subgroup of order 120 with index 10080 in $G$.

There are 63 such sets of six couples corresponding to the choices of $N^{(i j)}\left(\cong 4 \cdot S_{4}: 2\right)$ in $N$. Now we label each set of six couples corresponding to $N^{(i j)}$ by $i . j$. It is clear that $i . j$ has 6 (unordered) images under $N^{(i j)}$. Examples of set of six couples are

$$
\infty .11 \equiv\left\{\begin{array} { l } 
{ ( \infty , 1 1 ) , } \\
{ ( 1 , 1 ) , } \\
{ ( 0 , 3 ) , } \\
{ ( 0 , 3 ) , } \\
{ ( 1 0 , 1 3 ) , } \\
{ ( 0 1 , 3 1 ) , }
\end{array} \quad 2 . 1 3 \equiv \left\{\begin{array}{l}
(2,13), \\
(4,11), \\
(1,40), \\
(3,20), \\
(04,35), \\
(56,62) .
\end{array}\right.\right.
$$

3.3. The progenitor $2^{* 100}:\left(J_{2}: 2\right)$. A presentation for the progenitor is

$$
\begin{aligned}
\langle x, y, s, r, q| x^{2} & =y^{3}=(x y)^{8}=[x, y]^{4}=s^{2}=[s, y]=\left[s,[x, y]^{2}\right] \\
& =(x s)^{3}=\left(x y s^{x y x}\right)^{4}=r^{2}=[r, x]=[r, y]=(s r)^{3} \\
& \left.=q^{2}=[q, x]=[q, y]=[q, s]=1\right\rangle .
\end{aligned}
$$


TABLE 3.3. Double coset enumeration for $G_{2}(4): 2$.

\begin{tabular}{|c|c|c|}
\hline Label $[w]$ & Coset stabilizing subgroup $N^{(w)}$ & No. of cosets \\
\hline$[*]$ & $N$ & 1 \\
\hline$[\mathrm{x}]$ & $\begin{array}{l}N^{\mathrm{X}}=N^{(\mathrm{x})} \cong U_{3}(3): 2 \\
\text { with orbits } 1+36+63 \text { on the } 100 \text { points }\end{array}$ & 100 \\
\hline$[\mathrm{x} \infty]=[\mathrm{x}]$ & Since $\mathrm{x} \infty \mathrm{x} \sim * \Rightarrow \mathrm{x} \infty \sim \mathrm{x}$ & \\
\hline$[\mathrm{x} 0.5]$ & $\begin{array}{l}N^{\mathrm{x} 0.5}=\left\langle(0,5)(1,6)(2,3)(3,1)(4,4)(5,0)(6,2) s_{4} s_{4},\right. \\
(1,2)(3,6)(1,2)(3,6),(1,6)(2,3)(0,5)(1,2),(1,3) \\
\left.(2,6)(0,5)(3,6) s_{4} s_{5}\right\rangle \cong 2 \cdot S_{4}: 2 \text {. } \\
\text { Each element } i \text { of the } 12 \text {-orbits of } 2 \cdot S_{4}: 2 \text { is } \\
\text { joined to both of } \mathrm{x} \text { and } 0.5, \text { that is, } \mathrm{x} i \text { and } i 0.5 \\
\text { are special pairs } \Rightarrow N^{(\mathrm{x} 0.5)} \geq\left\langle\pi_{\infty 0.5}, \pi_{00.5}, \pi_{40.5}\right. \\
\pi_{50.5} \pi_{00.5}, \pi_{40.5}, \pi_{50.5}, \pi_{040.5}, \pi_{400.5}, \pi_{440.5}, \pi_{450.5} \\
\pi_{540.5},(1,3)(2,6)(0,5)(3.6) s_{4} S_{5},(0,5)(1,6)(2,3) \\
\left.(3,1)(4,4)(5,0)(6,2) s_{4} S_{4}\right\rangle \cong 2_{-}^{1+4} . S_{5}, \\
\text { with } 20+80 \text { orbits on } 100 \text { points }\end{array}$ & 315 \\
\hline$[\mathrm{x} 0.51]=[\mathrm{x} 0.5]$ & $\begin{array}{l}q_{\mathrm{x}} q_{0.5} q_{1}=q_{0.5} q_{\mathrm{x}} q_{1}=q_{0.5} \pi_{\mathrm{x} 1} q_{\mathrm{x}}=q_{0.5} r_{1} q_{\mathrm{x}} \sim q_{0.4} q_{\mathrm{x}} \\
\text { since } r_{0} r_{5} r_{1}=r_{0} r_{5} r_{1} r_{5} r_{5}=r_{0} \pi_{15} r_{5} \sim r_{4} r_{5} \sim r_{0} r_{4}\end{array}$ & \\
\hline
\end{tabular}

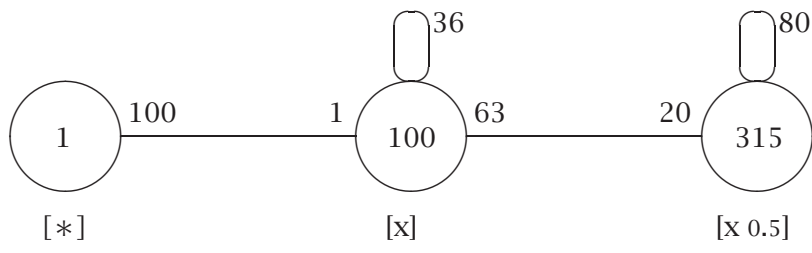

FIGURE 3.4

Here the symmetric generators are labeled with the vertices of the 100-point graph. A single vertex is labeled $\mathrm{x}, 36$ vertices are labeled $\infty, 0,1,2,3,4,5,6,0,1,2,3$, 4,5,6, 01, 02, 04, 10, 11, 13, 20, 22, 26, 31, 35, 36, 40, 44, 45, 53, 54, 56, 62, 63, and 65 and 63 vertices are labeled by the elements of the set of all six couples. We would like to know which elements of the control subgroup $N \cong J_{2}: 2$ can be written in terms of two symmetric generators. Lemma 2.3 says that $\mathscr{C}_{N}\left(N^{\mathrm{x} \infty}\right)=\langle r\rangle$. We make the assumption that $\pi_{\mathrm{x} \infty}(=r)=q_{\mathrm{x}} q_{\infty} q_{\mathrm{x}}$, a word in the symmetric generators $q_{\mathrm{x}}$ and $q_{\infty}$ of the shortest length that does not lead to collapse.

Consider the group

$$
G \cong \frac{2^{* 100}:\left(J_{2}: 2\right)}{\pi_{\mathrm{x} \infty}=q_{\mathrm{x}} q_{\infty} q_{\mathrm{x}}}
$$

The double cosets and coset stabilizing subgroups are shown in Table 3.3. The double coset enumeration yields a Cayley diagram of $G$ over $N$ (see Figure 3.4). 
The coset enumeration shows that the group defined by the symmetric presentation contains a homomorphic image of $N$ to index at most $1+100+315=416$, and gives a convenient name to each 416 cosets in terms of 100 symmetric generators. Moreover, the action of the generators on the 416 cosets, by right multiplication, is implicit in the enumeration and so it is readily checked that these permutations satisfy the given relation. Moreover, $G$ has order $\left|J_{2}: 2\right| \times 416=$ 503193600 , and $G$ is the automorphism group of a rank-3 graph (obtained from the above diagram by joining the coset $w$ to the coset $i w$ ) of valence 100 on 416 points in which the point stabilizer is $J_{2}: 2$. This is, of course, the group $G_{2}(4): 2$, see [2].

Every element of $G$ can be represented by a permutation on 100 letters (elements of $J_{2}: 2$ ) followed by a word in the symmetric generators of length at most two. Also each element of $G$ can be represented by the expression $\pi u v w$, where $\pi$ is a permutation on 14 letters (element of $L_{3}(2): 2$ ) and $u, v, w$ are words of length at most two in the symmetric generators $s$ 's, $r$ 's, and $q$ 's, respectively. We refer to this as nested symmetric representation of an element of the group. The (nested) symmetrically represented generators for each of the maximal subgroups of $G \cong\langle(0,0)(1,1)(2,2)(3,3)(4,4)(5,5)(6,6),(0,5,6)(1,2,4)$ $\left.(1,6,5)(2,4,3), s_{0}, r_{\infty}, q_{\mathrm{x}}\right\rangle$

$$
\begin{aligned}
G_{2}(4) \cong & \left\langle(0,0)(1,1)(2,2)(3,3)(4,4)(5,5)(6,6) s_{0} r_{\infty} q_{x},\right. \\
& (0,5,6)(1,2,4)(1,6,5)(2,4,3)\rangle
\end{aligned}
$$

is a simple group of index 2 in $G$;

$$
\begin{array}{r}
J_{2}: 2 \cong\langle(0,0)(1,1)(2,2)(3,3)(4,4)(5,5)(6,6), \\
\left.(0,5,6)(1,2,4)(1,6,5)(2,4,3), s_{0}, r_{\infty}\right\rangle
\end{array}
$$

is a subgroup of index 416 in $G$ and is the stabilizer of a point in the 416-point graph;

$$
2^{2+8}:\left(3 \times A_{5}\right): 2 \cong\left\langle(0,0)(1,1)(2,2)(3,3)(4,4)(5,5)(6,6) r_{2} r_{13} q_{6} q_{1.65}, s_{0}\right\rangle
$$

is a subgroup of index 1365 in $G$;

$$
\begin{aligned}
2^{4+6}:\left(A_{5} \times 3\right): 2 \cong & \left\langle(0,1,2,6,5,3,4)(0,2,3,6,5,4,1) r_{1} q_{1} q_{4.63},\right. \\
& \left.(0,1)(2,4,5,6)(0,4,3,2)(5,6) s_{6} s_{5} r_{2} r_{54} q_{3.53}\right\rangle
\end{aligned}
$$

is a subgroup of index 1365 in $G$;

$$
\begin{aligned}
& U_{3}(4): 4 \cong\left\langle(0,5,2)(3,6,4)(0,1,3)(2,6,4) s_{5} r_{26},\right. \\
&\left.(0,1,4)(2,5,3)(0,4,1)(2,3,5) s_{6} r_{22} q_{11}\right\rangle
\end{aligned}
$$


is a subgroup of index 2016 in $G$;

$$
\begin{aligned}
3 \cdot L_{3}(4): 2^{2} \cong & \left\langle(0,0)(1,1)(2,4)(3,3)(4,2)(5,6)(6,5) q_{\mathrm{x}},\right. \\
& (0,5)(1,6,2,3)(0,5)(1,3,2,6) s_{4}, \\
& \left.(0,5,5,0)(1,1,3,6,2,2,6,3)(4,4) r_{0} r_{5}\right\rangle
\end{aligned}
$$

is a subgroup of index 2080 in $G$ and is the normalizer of

$$
(0,0)(1,1)(2,2)(3,3)(4,4)(5,5)(6,6) s_{0},
$$

an element of order 3 in class $3 A$;

$$
\begin{array}{r}
U_{3}(3): 2 \times 2 \cong\langle(0,0)(1,1)(2,2)(3,3)(4,4)(5,5)(6,6), \\
\left.(0,5,6)(1,2,4)(1,6,5)(2,4,3), s_{0}, q_{x}\right\rangle
\end{array}
$$

is a subgroup of index 20800 in $G$ and is the centralizer of $q_{\mathrm{x}}$, an involution in class $2 C$;

$$
\begin{aligned}
\left(A_{5} \times A_{5}\right): 2 \cong & \left\langle(0,5,1,4,6,1,4,3)(2,6)(3,0,5,2) s_{0} r_{0} r_{2} q_{3},\right. \\
& \left.(0,4,3)(1,5,6)(0,6,2)(1,4,5) s_{4} s_{0} r_{1} r_{45} q_{5} q_{3.40}\right\rangle
\end{aligned}
$$

is a subgroup of index 69888 in $G$; and

$$
\begin{aligned}
L_{2}(13): 2 \cong\langle & (0,4,4,0,2,2)(1,6,6,1,5,5)(3,3) s_{0} r_{\infty} q_{x}, \\
& \left.(0,6)(1,4)(1,5)(3,4) q_{0.6}\right\rangle
\end{aligned}
$$

is a subgroup of index 230400 in $G$.

3.4. The progenitor $2^{* 416}:\left(G_{2}(4): 2\right)$. A presentation for the progenitor is

$$
\begin{aligned}
\langle x, y, s, r, q, p| x^{2} & =y^{3}=(x y)^{8}=[x, y]^{4}=s^{2}=[s, y]=\left[s,[x, y]^{2}\right] \\
& =(x s)^{3}=\left(x y s^{x y x}\right)^{4}=r^{2}=[r, x]=[r, y]=(s r)^{3} \\
& =q^{2}=[q, x]=[q, y]=[q, s]=(r q)^{3}=[p, x] \\
& =[p, y]=[p, s]=[p, r]=1\rangle .
\end{aligned}
$$

Here the symmetric generators which correspond to the vertices of the 416point graph are denoted by $0,1, \ldots, 415$, where $p_{0}=p_{q_{*}}, p_{1}=p_{q_{\mathrm{x}}}$, and $p_{2}=$ $p_{q_{\infty}}, \ldots$. We would like to know which elements of the control subgroup $N \cong$ $G_{2}(4): 2$ can be written in terms of two symmetric generators. Lemma 2.3 states that $\mathscr{C}_{N}\left(N^{01}\right)=\langle q\rangle$. We make the assumption that $\pi_{01}(=q)=p_{0} p_{1} p_{0}$, a word in the symmetric generators $p_{0}$ and $p_{1}$ of the shortest length that does not lead to collapse.

A nice way of looking at Suz : 2 is the way Suzuki constructed the group [2] as a rank-3 extension of $G_{2}(4): 2$ of degree 1782 with suborbit sizes 1,416 , and 1365 . We will be looking at the group from this point of view to identify our homomorphic image of the progenitor with Suz $: 2$. 
TABLE 3.4. Double coset enumeration for $3 \cdot$ Suz $: 2$.

\begin{tabular}{|c|c|c|}
\hline Label $[w]$ & Coset stabilizing subgroup $N^{(w)}$ & No. of cosets \\
\hline$[*]$ & $N$ & 1 \\
\hline [0] & $\begin{array}{l}N^{0}=N^{(0)} \cong J_{2}: 2, \\
\text { with orbits } 1+100+315 \text { on the } 416 \text { points }\end{array}$ & 416 \\
\hline$[01]=[0]$ & Since $010 \sim * \Rightarrow 01 \sim 1,0$ is an element of the 100 -orbit & \\
\hline$[0 i]$ & $\begin{array}{l}N^{0 i} \cong 2_{-}^{1+4}: S_{5}, i \text { is an element of the } 315 \text {-orbit, } \\
\left|N^{(0 i)}\right|=32 \times\left|\operatorname{Stab}_{N^{(0 i)}}(0)\right| \geq 32 \times 3840 . \\
N^{(0 i)} \cong 2^{2+8}: S_{5}, \text { thus the double coset }[0 i] \text { has } \\
\text { at most } 4095 \text { single cosets, } N^{(0 i)} \text { has orbits } \\
32+320+64 \text { on the } 416 \text { points }\end{array}$ & 4095 \\
\hline$[0 i l]=[0 i]$ & $\begin{array}{l}\text { Dil } \sim 0 \pi_{i l} i \sim 0^{\pi_{i l}} i, i \text { joined to } l, l \text { is an element } \\
\text { of } 320 \text {-orbit }\end{array}$ & \\
\hline$[0 i j]$ & $\begin{array}{l}N^{0 i j} \cong 2_{-}^{1+4}: A_{5}, j \text { is an element of the } 64 \text {-orbit since } \\
N^{0 i j} \text { is maximal in } J_{2} \text {, adding any permutation of } N \\
\text { which fixes the coset } N p_{0} p_{i} p_{j} \text { to } N^{0 i j} \Rightarrow N^{(0 i j)} \cong J_{2} \text {, } \\
\text { with orbits } 315+100+1 \text { on the } 416 \text { points }\end{array}$ & 832 \\
\hline$[0 i j l]=[0 i j]$ & $\begin{array}{l}0 i j l \sim 0 i \pi_{j l} j \sim(0 i)^{\pi} j l j, j \text { joined to } l, l \text { is an element } \\
\text { of } 100 \text {-orbit }\end{array}$ & \\
\hline$[0 i j k]$ & $\begin{array}{l}N^{0 i j k} \text { is maximal in } G_{2}(4) \text {, adding any permutation } \\
\text { of } N \text { which fixes the coset } N p_{0} p_{i} p_{j} p_{k} \text { to } \\
N^{0 i j k} \Rightarrow N^{(0 i j k)} \cong G_{2}(4) \text {, which is transitive on the } \\
\text { symmetric generators }\end{array}$ & 2 \\
\hline
\end{tabular}

Consider the group

$$
G \cong \frac{2^{* 416}:\left(G_{2}(4): 2\right)}{\pi_{01}=p_{0} p_{1} p_{0}}
$$

The double cosets and coset stabilizing subgroups are given in Table 3.4. The double coset enumeration shown in Table 3.4 yields a Cayley diagram of $G$ over $N$ (see Figure 3.5).

The coset enumeration shows that the group defined by the symmetric presentation contains a homomorphic image of $N$ to index at most $(1+416+$ $4095+832+2)=5346$, and gives a convenient name to each 5346 cosets in terms of 416 symmetric generators. Moreover, the action of the generators on the 5346 cosets, by right multiplication, is implicit in the enumeration and so it is readily checked that these permutations satisfy the given relation. Thus $|G|=\left|G_{2}(4): 2\right| \times 5346=2690072965600$, and $G$ is isomorphic to the group $3 \cdot$ Suz : 2. Finally, adding the relator $\left(\pi p_{0}\right)^{13}$ [2], where $\pi$ induces a permutation of cyclic shape $1 \cdot 3 \cdot 4 \cdot 6^{2} \cdot 12^{33}$ on 416 letters, to those of $G$ gives Suz : 2. Again the double coset enumeration over $N$ gives a Cayley diagram (see Figure 3.6). 


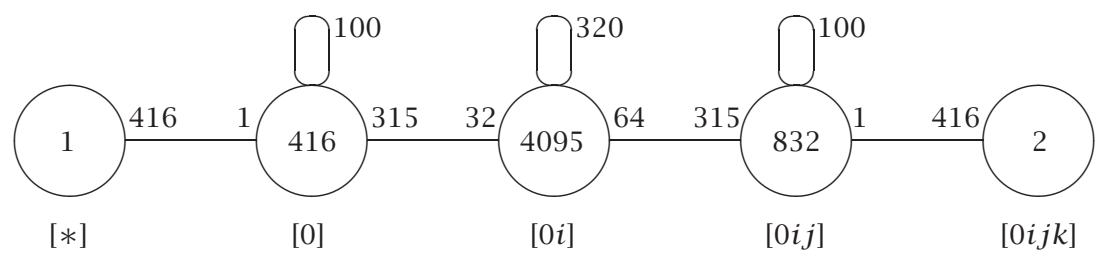

FIGURE 3.5

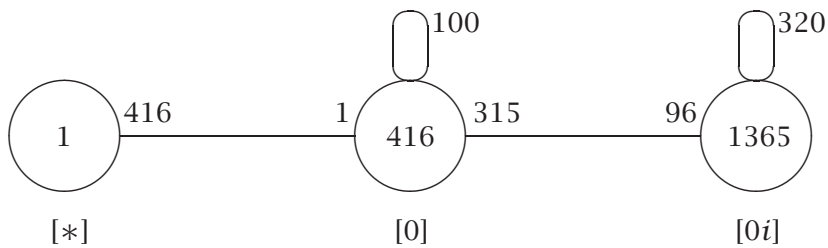

FIGURE 3.6

Every element of $G(\cong$ Suz : 2$)$ can be represented by a permutation on 416 letters (elements of $\left.G_{2}(4): 2\right)$ followed by a word in the symmetric generators of length at most two. Also each element of $G$ can be represented by the expression $\pi u v w z$, where $\pi$ is a permutation on 14 letters (element of $L_{3}(2): 2$ ) and $u, v, w$, and $z$ are words of length at most two in the symmetric generators $s$ 's, $r$ 's, $q$ 's, and $p$ 's, respectively. We refer to this as nested symmetric representation of an element of the group. The (nested) symmetrically represented generators for each of the maximal subgroups of $G \cong$ $\left\langle(0,0)(1,1)(2,2)(3,3)(4,4)(5,5)(6,6),(0,5,6)(1,2,4)(1,6,5)(2,4,3), s_{0}, r_{\infty}, q_{x}, p_{0}\right\rangle$

$$
\begin{aligned}
\text { Suz } \cong & \left\langle(0,0)(1,1)(2,2)(3,3)(4,4)(5,5)(6,6) s_{0},\right. \\
& (0,0)(1,1)(2,2)(3,3)(4,4)(5,5)(6,6) r_{\infty}, \\
& (0,0)(1,1)(2,2)(3,3)(4,4)(5,5)(6,6) q_{x}, \\
& (0,0)(1,1)(2,2)(3,3)(4,4)(5,5)(6,6) p_{0}, \\
& (0,5,6)(1,2,4)(1,6,5)(2,4,3)\rangle
\end{aligned}
$$

is a subgroup of index 2 in $G$;

$$
\begin{aligned}
G_{2}(4): 2 \cong & \langle(0,0)(1,1)(2,2)(3,3)(4,4)(5,5)(6,6), \\
& \left.(0,5,6)(1,2,4)(1,6,5)(2,4,3), s_{0}, r_{\infty}, q_{\mathrm{x}}\right\rangle
\end{aligned}
$$

is a subgroup of index 1782 in $G$ and is the stabilizer of a point in the 1782point graph;

$$
3 U_{4}(3):\left(2^{2}\right)_{133} \cong\left\langle(0,6,3,5)(2,4)(1,3)(2,5,6,4) s_{0} s_{2} r_{0} r_{6} p_{0}, s_{0} q_{\mathrm{x}}\right\rangle
$$


is a subgroup of index 22880 in $G$ and is the normalizer of

$$
(0,0)(1,1)(2,2)(3,3)(4,4)(5,5)(6,6) s_{0},
$$

an element of order 3 in class $3 A$;

$$
2_{-}^{1+6} \cdot U_{4}(2): 2 \cong\left\langle(0,0)(1,2)(2,1)(3,6)(4,4)(5,5)(6,3) s_{0} q_{\mathrm{x}}, s_{4} r_{\infty} p_{0}\right\rangle
$$

is a subgroup of index 135135 in $G$ and is the normalizer of $(1,2)(3,6)(1,2)(3,6)$, an involution in class $2 A$;

$$
\begin{aligned}
J_{2}: 2 \times 2 \cong & \langle(0,0)(1,1)(2,2)(3,3)(4,4)(5,5)(6,6), \\
& \left.(0,5,6)(1,2,4)(1,6,5)(2,4,3), s_{0}, r_{\infty}, p_{0}\right\rangle
\end{aligned}
$$

is a subgroup of index 370656 in $G$ and is the centralizer of $p_{0}$, an involution in class $2 C$; and

$$
\begin{aligned}
M_{12}: 2 \times 2 \cong & \left\langle(0,6)(1,0,4,5,6,3)(2,4,3,2,5,1) r_{5} r_{4} q_{x} q_{4.5} p_{0},\right. \\
& \left.(1,6)(2,3)(0,5)(1,2) q_{\infty} p_{0},(1,4)(3,5)(1,4)(3,5) s_{6} r_{0}\right\rangle
\end{aligned}
$$

is a subgroup of index 2358720 in $G$ and is the centralizer of

$$
(0,6)(1,5)(2,2)(3,1)(4,3)(5,4)(6,0) r_{\infty} p_{0},
$$

an involution in class 3D.

3.5. The progenitor $\left(2^{2}\right) * 1782:(3 \cdot$ Suz $: 2)$. A presentation for the progenitor is

$$
\begin{aligned}
\langle x, y, s, r, q, p, t| x^{2} & =y^{3}=(x y)^{8}=[x, y]^{4}=s^{2}=[s, y]=\left[s,[x, y]^{2}\right] \\
& =(x s)^{3}=\left(x y s^{x y x}\right)^{4}=r^{2}=[r, x]=[r, y] \\
& =(s r)^{3}=q^{2}=[q, x]=[q, y]=[q, s]=(r q)^{3} \\
& =[p, x]=[p, y]=[p, s]=[p, r]=(q p)^{3} \\
& =[t, x]=[t, y]=[t, s]=[t, q]=1\rangle .
\end{aligned}
$$

We seek a monomial semilinear 1782-dimensional representation of $N \cong 3$. Suz : 2 over $\mathrm{GF}_{4}$, the Galois field of order 4 . Elements in $3 \cdot$ Suz : 2 can thus act as permutations of 1782 Klein four-groups, followed by the field automorphism $\sigma$ of $\mathrm{GF}_{4}$. The cyclic groups $T_{i}$ are replaced by copies of the Klein four-groups which we label by $V_{i}=\left\langle t_{i_{1}}, t_{i_{2}} t_{i_{3}}\right\rangle$. The centralizer in $N$ of $V_{1}$ is isomorphic to $G_{2}(4)$ with orbits $1+416+1365$. Centralizing a further four-group in the 416-orbit, say $V_{2}$, yeilds a subgroup isomorphic to $J_{2}$ whose centralizer in $N$ 


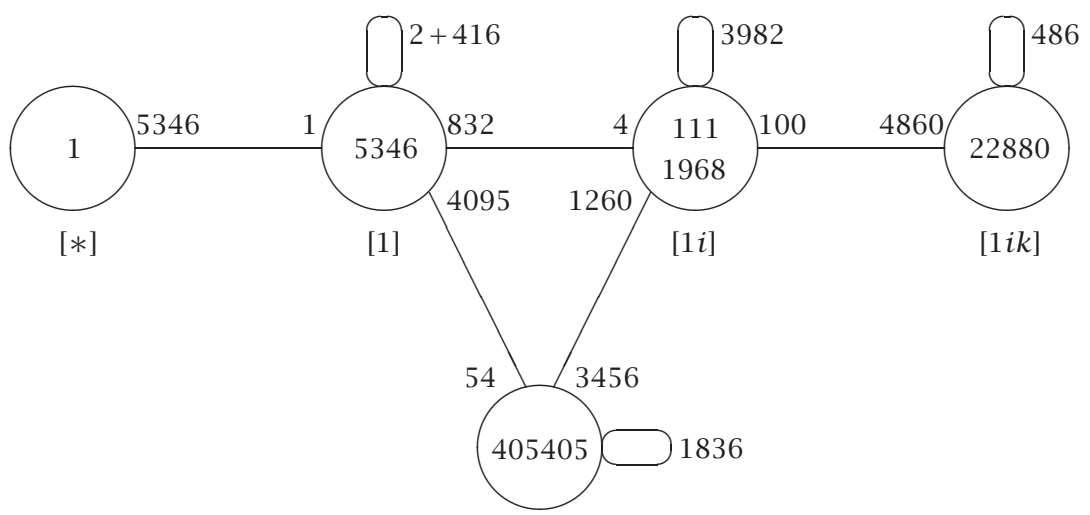

$[1 j]$

FIGURE 3.7

is a copy of $S_{3}$ :

$$
\left\langle V_{1}, V_{2}\right\rangle \cap N \leq \mathscr{C}_{N}\left(N^{12}\right) \cong S_{3}
$$

Elements of order 3 in $S_{3}$ cycle the involutions in each of the two fixed fourgroups, while its involutions interchange them and apply the field automorphism $\sigma$. We thus seek an image of $\left(2^{2}\right)^{* 2}: S_{3}$, where

$$
S_{3} \cong\left\langle a=\left(\begin{array}{cc}
\omega & \cdot \\
\cdot & \omega
\end{array}\right), b=\left(\begin{array}{ll}
\cdot & 1 \\
1 & \cdot
\end{array}\right) \sigma\right\rangle .
$$

Factoring this by the relator $\left(b t_{1_{1}}\right)^{3}$ is easily seen to give the image $A_{5}$. For example, if $a=(3,4,5), b=(1,2)(4,5), t_{1_{1}}=(1,3)(4,5)$, then

$$
\begin{array}{ll}
t_{1_{1}}=(1,3)(4,5), & t_{2_{1}}=(2,3)(4,5), \\
t_{1_{2}}=\omega t_{1_{1}}=(1,4)(3,5), & t_{2_{2}}=\omega t_{2_{1}}=(2,4)(3,5), \\
t_{1_{3}}=\bar{\omega} t_{1_{1}}=(1,5)(3,4), & t_{2_{3}}=\bar{\omega} t_{2_{1}}=(2,5)(3,4) .
\end{array}
$$

If the progenitor $\left(2^{2}\right)^{* 1782}: 3 \cdot$ Suz $: 2$ is factored by a corresponding relation, we obtain

$$
\mathrm{Co}_{1} \cong \frac{\left(2^{2}\right)^{* 1782}:(3 \cdot \mathrm{Suz}: 2)}{\left(\left(\begin{array}{l}
\cdot 1 \\
1 \cdot
\end{array}\right) \sigma t_{1_{1}}\right)^{3}} .
$$

The central element of order three [2], which fixes each of the 1782 four-groups while cycling its nontrivial element, is $\left([x, y]^{2}\left([x, y]^{2}\right)^{y} x\left([x, y]^{2}\right)^{y} x \operatorname{srqp}\right)^{13}$. With the help of the program in [5], the enumeration over $N$ gives a Cayley diagram (see Figure 3.7). 


\section{REFERENCES}

[1] W. Bosma, J. Cannon, and C. Playoust, The Magma algebra system. I. The user language, J. Symbolic Comput. 24 (1997), no. 3-4, 235-265.

[2] J. H. Conway, R. T. Curtis, S. P. Norton, R. A. Parker, and R. A. Wilson, Atlas of Finite Groups, Oxford University Press, Eynsham, 1985.

[3] R. T. Curtis, Natural constructions of the Mathieu groups, Math. Proc. Cambridge Philos. Soc. 106 (1989), no. 3, 423-429.

[4] Symmetric presentations. I. Introduction, with particular reference to the Mathieu groups $M_{12}$ and $M_{24}$, Groups, Combinatorics \& Geometry (Durham, 1990), London Math. Soc. Lecture Note Ser., vol. 165, Cambridge University Press, Cambridge, 1992, pp. 380-396.

[5] M. Sayed, Computational methods in symmetric generation of groups, Ph.D. thesis, University of Birmingham, 1998.

[6] L. H. Soicher, Presentations for Conway's group $\mathrm{Co}_{1}$, Math. Proc. Cambridge Philos. Soc. 102 (1987), no. 1, 1-3.

[7] M. Suzuki, Group Theory. I, Grundlehren der mathematischen Wissenschaften, vol. 247, Springer-Verlag, Berlin, 1982.

[8] R. A. Wilson, The complex Leech lattice and maximal subgroups of the Suzuki group, J. Algebra 84 (1983), no. 1, 151-188.

[9] _ The maximal subgroups of Conway's group $\mathrm{Co}_{1}$, J. Algebra 85 (1983), no. 1, 144-165.

Mohamed Sayed: Department of Mathematics and Computer Science, Faculty of Science, Kuwait University, P.O. Box 5969, Safat 13060, Kuwait

E-mail address: msayed@mcs.sci . kuniv.edu.kw 


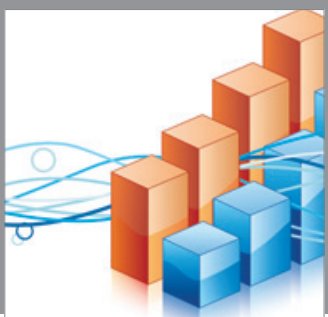

Advances in

Operations Research

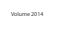

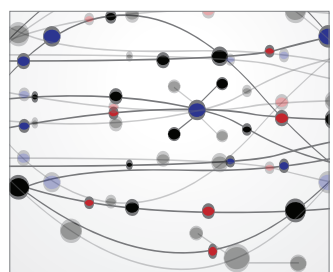

\section{The Scientific} World Journal
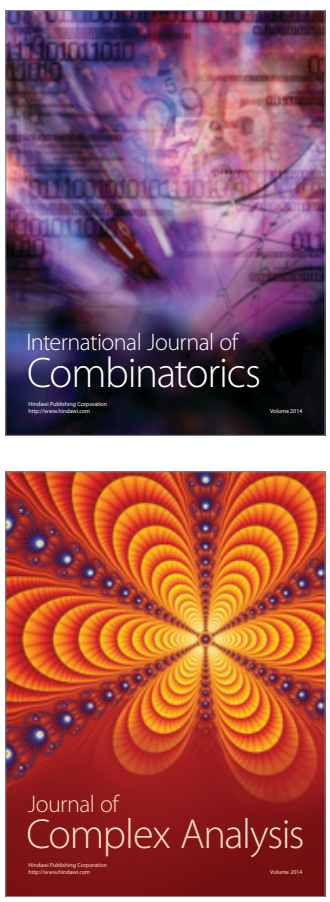

International Journal of

Mathematics and

Mathematical

Sciences
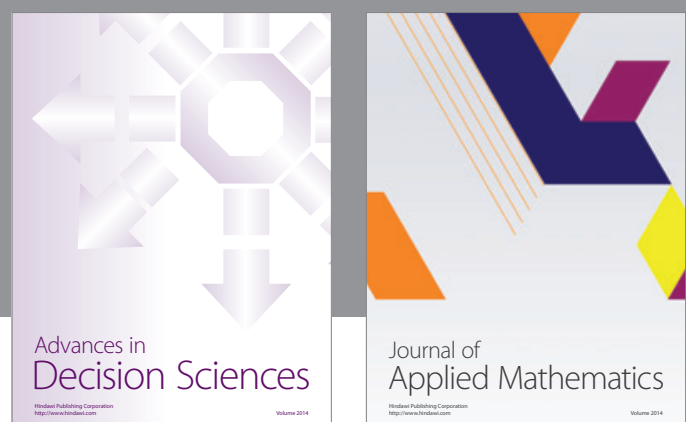

Journal of

Applied Mathematics
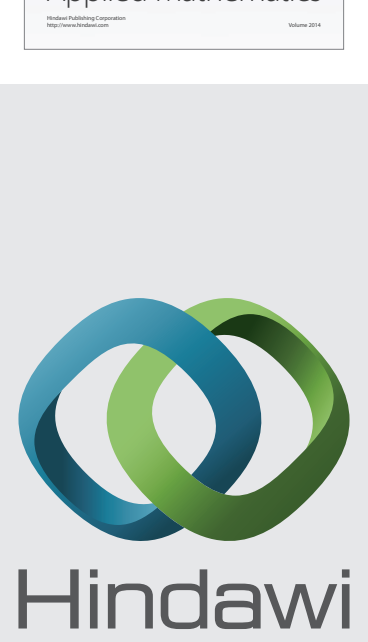

Submit your manuscripts at http://www.hindawi.com
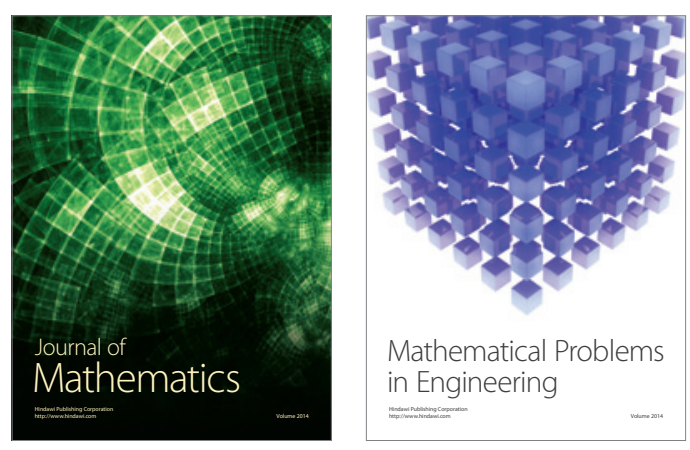

Mathematical Problems in Engineering
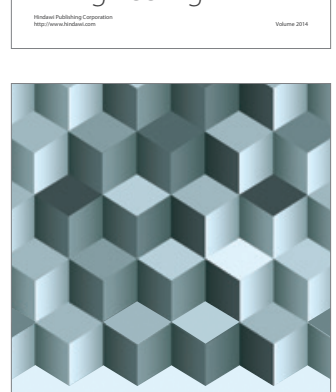

Journal of

Function Spaces
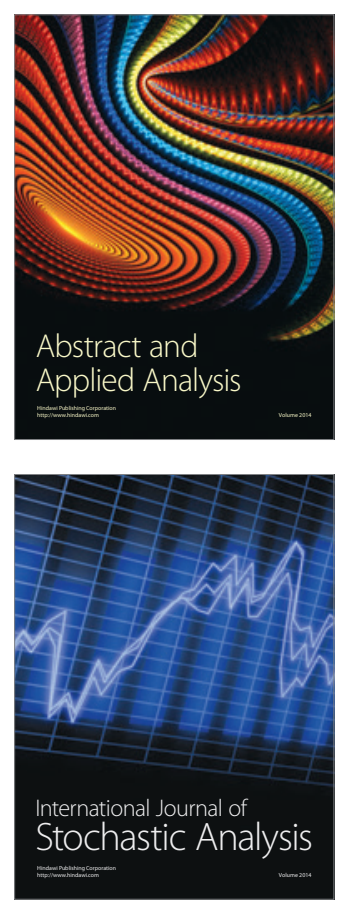

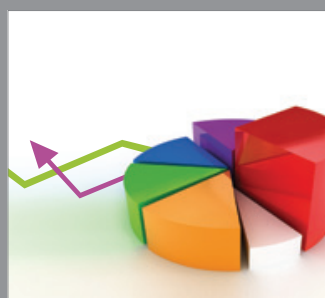

ournal of

Probability and Statistics

Promensencen
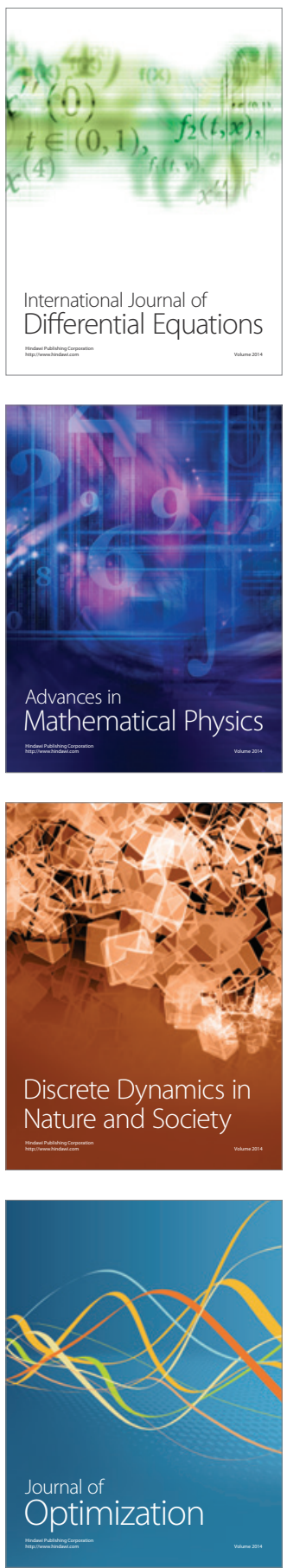